\title{
Time variable funnel flows
}

\author{
Silvia H. P. Alencar ${ }^{1}$ \\ ${ }^{1}$ Departamento de Física - ICEx - UFMG, CP 702, 31270-901, Belo Horizonte, MG, Brazil \\ email: silvia@fisica.ufmg.br
}

\begin{abstract}
Magnetospheric accretion models are the current consensus to explain the main observed characteristics of classical T Tauri stars. In recent years the concept of a static magnetosphere has been challenged by synoptic studies of classical $\mathrm{T}$ Tauri stars that show strong evidence for the accretion process to be dynamic on several timescales and governed by changes in the magnetic field configuration. At the same time numerical simulation results predict evolving funnel flows due to the interaction between the stellar magnetosphere and the inner disk region. In this contribution we will focus on the main recent observational evidences for time variable funnel flows and compare them with model predictions.
\end{abstract}

Keywords. Stars: pre-main-sequence, stars: magnetic fields, circumstellar matter, accretion.

\section{From static to dynamical models}

Young solar-type stars (T Tauri stars, TTS) typically accrete material from their circumstellar disks for a few million years. Accretion is thought to be the source of the powerful jets observed in young stars and has a deep impact on the evolution of low mass stars by providing mass and angular momentum and by influencing disk evolution at a time when planets are forming. Understanding the accretion process in TTS is therefore an important issue for star and planet formation theories.

Magnetospheric accretion models (Hartmann, Hewett \& Calvet 1994; Shu, Najita, Ostriker, et al. 1994; Muzerolle, Hartmann \& Calvet 2001; Kurosawa, Harries \& Symington 2006) are the current consensus to describe the main observational characteristics of accreting TTSs (classical T Tauri stars, CTTSs). In these models, accretion from the disk to the star is mediated by the stellar magnetic field that interacts with the inner circumstellar disk. The inner disk is truncated at a point where the magnetic pressure overcomes the ram pressure due to accretion and the accreting material is channeled to the star following magnetic field lines at near free-fall velocities. As the accreting material hits the star, a shock is formed at the stellar surface and a hot continuum, known as veiling, is emitted. Stellar and disk winds may be present in the models, and help drive away a magnetically driven outflow. The general characteristics of magnetospheric accretion models are supported by observational results. Magnetic fields of 1 to $3 \mathrm{kG}$ have been measured at the surface of T Tauri stars (Johns-Krull, Valenti, Hatzes, et al. 1999; Valenti \& Johns-Krull 2004; Symington, Harries, Kurosawa, et al. 2005), which are strong enough to disrupt the inner disk and channel magnetospheric accretion in funnel flows. Accretion columns are inferred through the common observation of inverse $\mathrm{P}$ Cygni profiles with redshifted absorptions reaching several hundred $\mathrm{km} \mathrm{s}^{-1}$, which indicates that gas is accreted onto the star from a distance of a few stellar radii (Edwards, Hartigan, Ghandour, et al. 1994). Accretion column evidence also comes from the successful modeling of strong optical and IR emission lines that are formed, at least in part, in the magnetospheric accretion flow (Hartmann et al. 1994; Muzerolle et al. 2001). Evidence of accretion shocks at the stellar surface comes from the successful fitting of the UV and optical excesses observed in CTTSs by accretion shock models (Calvet \& 
Gullbring 1998; Gullbring, Calvet, Muzerolle, et al. 2000) and from the analysis of light curve variability due to hot spots at the stellar surface (Bouvier, Covino, Kovo, et al. 1995). Numerical simulations of the magnetic star-disk interaction have also given support to the general magnetospheric accretion scenario. Starting from a large-scale dipolar magnetosphere configuration, simulation results show that an inner magnetospheric cavity develops and material is accreted to the star through funnel flows. As the accreting material hits the star, a hot spot or ring is formed, as predicted. The structure of funnel flows and hot spots depends on the accretion rate, on the topology of the magnetosphere and its inclination (Romanova, Ustyugova, Koldoba, et al. 2002; Romanova, Ustyugova, Koldoba, et al. 2003).

Although both observations and numerical simulations give a good support to the general predictions of magnetospheric accretion models, some problems with the standard models still remain. Most magnetospheric accretion models assume an axisymmetric configuration for the stellar magnetosphere. Observational results have shown however that the stellar magnetic axis can be inclined with respect to the rotation axis, causing periodical modulation of accretion and outflow diagnostics. Johns \& Basri (1995) have shown that the CTTS SU Aur presents inflow and outflow signatures that vary periodically at the stellar rotation period but are enhanced at opposite phases. They interpreted their results as due to an inclined dipole field with respect to the rotation axis that favors accretion and outflow $180^{\circ}$ out of phase. These results were later confirmed by Petrov, Gullbring, Ilyin, et al. (1996) with observations separated by several years from those analysed by Johns \& Basri (1995). Rotational modulation by hot spots (Bouvier et al. 1995) is also expected to arise due to an inclined magnetosphere, since an axisymmetric magnetospheric configuration would produce a uniform hot ring at the stellar surface whose emission would not vary as the star rotates. Rotational modulation by hot spots will also cause periodic veiling variations that are observed in CTTS systems (Bouvier, Grankin, Alencar, et al. 2003; Bouvier, Alencar, Boutelier, et al. 2007). Evidence for inclined magnetospheres also comes from the rotational modulation of the circular polarization signal measured in the HeI $5876 \AA$ line of several CTTSs (Valenti \& Johns-Krull 2004). Very recently, the first emission line profiles were calculated based on 3D MHD simulation results of Romanova et al. (2003) and Romanova, Ustyugova, Koldoba, et al. (2004b), who considered accretion onto a CTTS with a misaligned dipole with respect to the rotation axis. Kurosawa, Romanova \& Harries (2007) took the density, velocity and temperature structures of the MHD simulations and used them to calculate the rotationally induced line variability of several emission lines $(\mathrm{H} \beta, \mathrm{Pa} \beta$ and $\mathrm{Br} \gamma)$ that are commonly observed in CTTSs. This will allow for the first time a direct comparison between the emission line profiles originating from a complex magnetospheric structure and the time variable observed line profiles of several CTTSs.

Another common assumption of standard magnetospheric accretion models is steadystate accretion. The variability observed on a timescale of hours in emission lines of CTTSs such as TW Hya (Alencar \& Batalha 2002), which is seen almost pole-on and is therefore not subjected to rotational modulation effects, seems to rule out steadystate accretion as a straightforward assumption. Overall the accretion process appears to be variable on several timescales, in hours due to non-steady accretion, like the observed veiling changes of RU Lup that varies during a single night (Stempels \& Piskunov 2002), in days due to rotational modulation effects caused by an inclined magnetosphere, like the periodical variability of the redshifted absorption component of $\mathrm{H} \beta$ in $\mathrm{SU}$ Aur (Johns \& Basri 1995), in weeks due to global instabilities of the stellar magnetosphere caused by differential rotation between the star and the disk, as discussed in the following 

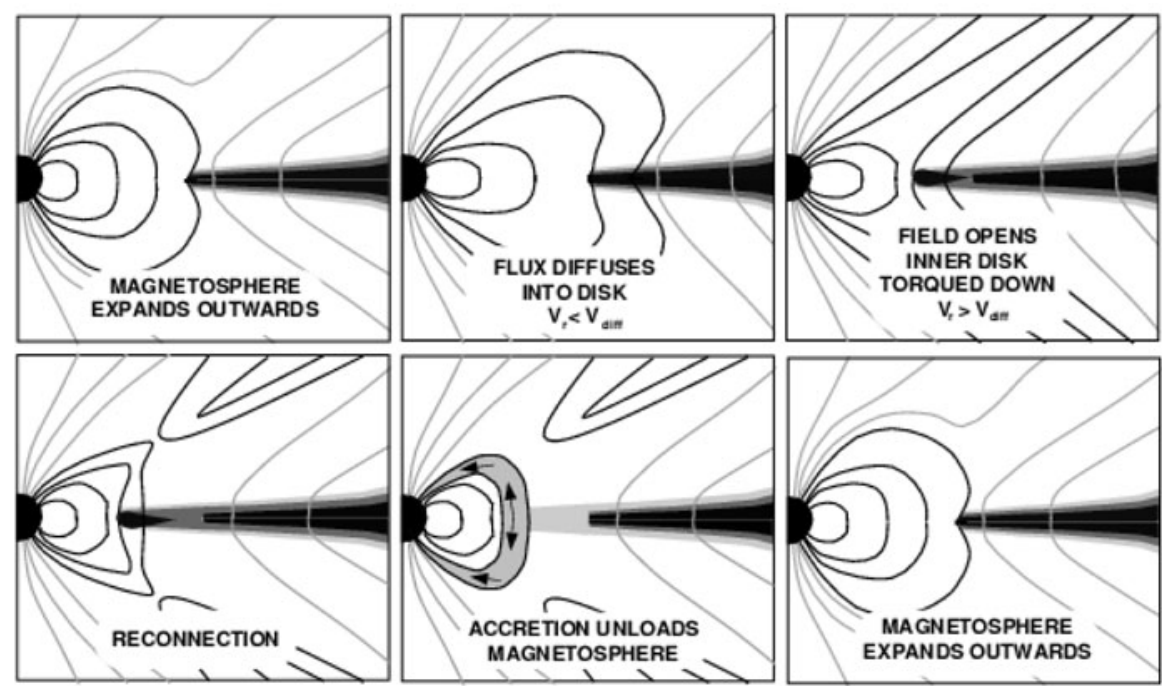

Figure 1. Differential rotation along the field lines leads to their expansion, opening and reconnection. From Goodson \& Winglee (1999).

paragraphs, and in years in EXor and FUor like events (Herbig 2007; Reipurth \& Aspin 2004).

Most magnetospheric accretion models assume a static magnetospheric configuration, where the stellar magnetic field may interact with the disk over a significant region. However, it is then expected that many stellar field lines will thread the disk at points that rotate differentially from the star. As the system rotates, this will give rise to magnetic field lines distorted by differential rotation, as commonly observed in numerical simulations. Some numerical simulations of the star-disk interaction predict that differential rotation between the star and the inner disk regions where the stellar magnetosphere is anchored, leads to the field lines expansion, opening and reconnection, which eventually restores the initial magnetospheric configuration (Fig. 1; Goodson, Winglee \& Böhm 1997; Goodson \& Winglee 1999; Matt, Goodson, Winglee, et al. 2002; Uzdensky, Königl \& Litwin 2002; Romanova, Ustyugova, Koldoba, et al. 2004a; von Rekowski \& Brandenburg 2004). Magnetospheric inflation cycles are expected by most numerical models to develop in a few Keplerian periods at the inner disk, and be accompanied by strong periodic outflow events, as reconnection takes place, and by time dependent accretion onto the star. The timescale of field line inflation is actually determined by the diffusion of the magnetic flux through the inner disk regions, which depends on the magnetic diffusivity in the disk that is unfortunately an uncertain parameter to calculate from first principles.

Most magnetospheric accretion models also assume a magnetosphere with a dipolar field geometry. Recently several observational results have indicated that the surface magnetic fields of young low-mass stars may be very complex and include high order multipoles (Valenti \& Johns-Krull 2004; Jardine, Cameron, Donati, et al. 2006; Daou, Johns-Krull \& Valenti 2006; Donati, Jardine, Petit, et al. 2007). Accretion may then eventually proceed through multipole filed lines if the interaction region between star and disk is not very far away from the stellar surface for only the dipole component to be important. This is more likely to happen in stars that present high accretion rates, since in that case the inner gas disk will extend closer to the star than in the low mass accretion rate systems. Multipolar axisymmetric fields were recently modeled by von Rekowski \& Brandenburg (2006). Their time-dependent simulations include a dynamo 
generated field in the star and the disk and results in a very complex and variable field configuration. In their simulations accretion occurs preferentially at low latitudes and is no longer periodic, as observed in the case of a stellar dipole field, but tends to be irregular and episodic. Recent MHD simulations by Long, Romanova \& Lovelace (2007) of an initial magnetosphere configuration composed of a quadrupole or of a dipole added to quadrupole field have shown that, as time evolves, the magnetospheric cavity, hot spots and field line inflation are still observed. The size and shape of the various features related to accretion (hot spots, light curves, magnetosphere dynamics) are different in each case, and accretion tends to proceed through field lines that reach the star closer to the equator than the poles.

The star-disk coupling is also affected by the field line dynamical opening. It becomes much less effective and then may not be able to explain alone the low rotational velocities observed in CTTSs (Matt \& Pudritz 2005a; von Rekowski \& Brandenburg 2006). The main exception among magnetospheric accretion models is the $\mathrm{X}$-wind model of Shu \& Shang (1997) that assumes the star-disk interaction to occur in a very small region close to the co-rotation point. The magnetic field lines that connect the star to the disk are also expected to inflate and reconnect in that model, but the departure from corotation is on average very small and the long-term interaction between the star and the disk can be analysed as a steady state of corotation. Therefore the angular momentum transport between the star and the disk is not much affected by the opening of the field lines in the $\mathrm{X}$-wind model. Most models however do have an angular momentum problem and stellar winds powered by accretion were suggested to help regulate the stellar angular momentum of accreting T Tauri stars (Matt \& Pudritz 2005b; Kwan, Edwards \& Fischer 2007; see also the contributions of Suzan Edwards and Sean Matt in this volume). To better understand the star-disk interaction it is therefore important to look for observational evidence of magnetic field line opening in CTTS systems.

Observational results have in recent years started to confirm the time-dependent nature and the many predictions of numerical simulations of the star-disk interaction. Episodic high velocity outbursts, possibly related to magnetospheric reconnection, have been reported for a few systems based on the velocity variation of blueshifted absorption components in emission line profiles on a timescale of hours to days (Alencar, Johns-Krull \& Basri 2001; Ardila, Basri, Walter, et al. 2002; Oliveira, Foing, van Loon, et al. 2000). Possible evidence for twisted magnetic field lines due to differential rotation between the star and the disk was proposed for SU Aur by Oliveira et al. (2000). A time delay of a few hours was measured between the appearance of high velocity redshifted absorption components in line profiles formed at different altitudes in the accretion column. This was interpreted as the crossing of an azimuthally twisted accretion column on the line of sight. Another possible evidence for magnetic field lines being twisted by differential rotation between the star and the disk and leading to quasi-periodic reconnection events was reported for the embedded protostellar source YLW 15, based on the observations of quasi-periodic X-ray energetic flares coming from large plasma-confining loops (loop length of $\sim 14 \mathrm{R}_{\odot} \sim 3 \mathrm{R}_{\star}$, Montmerle, Grosso, Tsuboi, et al. (2000) and Fig. 2). Evidence for very large flaring structures (much larger than the stellar radius), likely coming from reconnection events of magnetic loops that link the young stars to their disks, has also been reported by Favata, Flaccomio, Reale, et al. (2005) for about 15 stars in Orion based on the analysis of some of the most energetic X-ray flares observed in the Chandra Orion Ultradeep Project.

There is nowadays observational evidence that accretion and wind activity are nonsteady processes acting in the star-disk systems throughout the lifetime of accretion disks, which in certain cases may extend up to $\sim 10$ Myr (Jayawardhana, Coffey, Scholz 

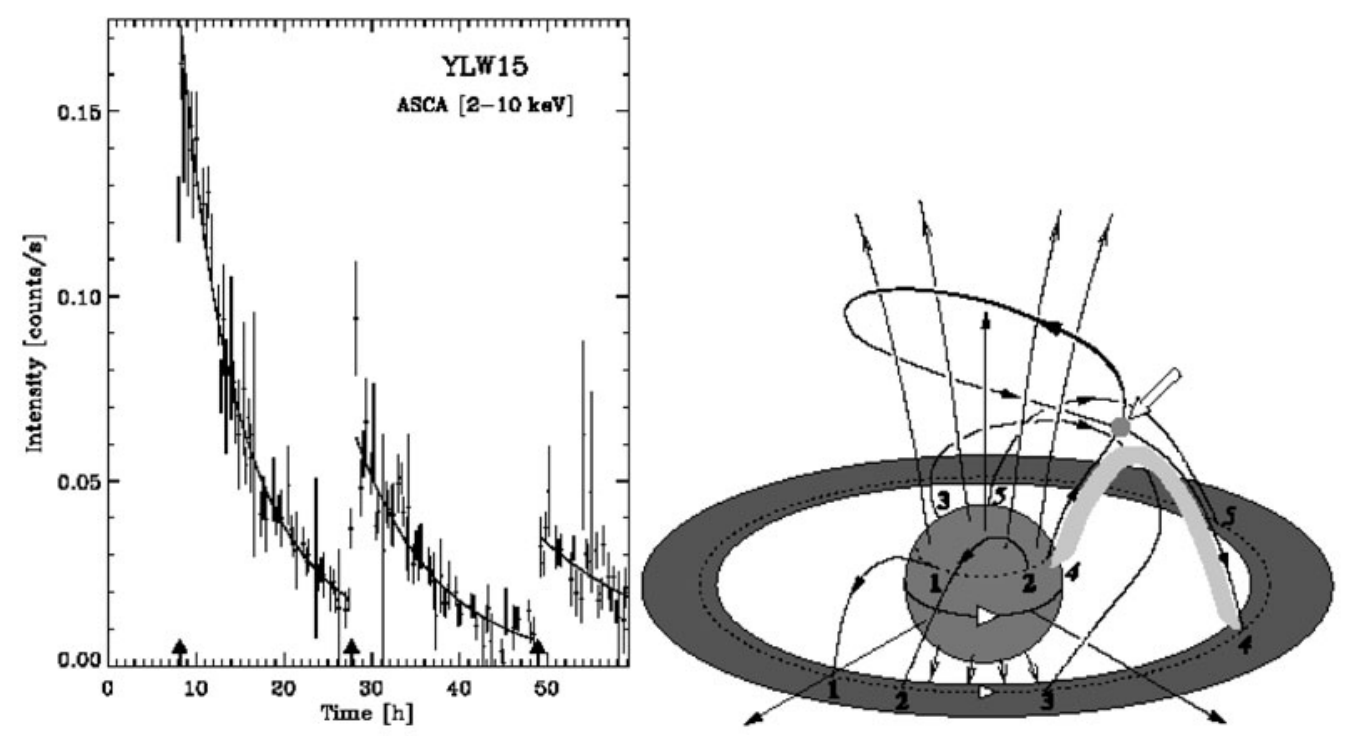

Figure 2. Left: light curve of YLW 15 obtained with ASCA (Tsuboi, Imanishi, Koyama, et al. 2000) showing three energetic flare events. Right: sketch of a plausible evolution of magnetic field configuration leading to reconnection and flaring as observed in the light curve of YLW 15. Figures from Montmerle et al. (2000).

et al. 2006). There is also recent observational evidence for time variable magnetospheric accretion onto brown dwarfs (Scholz \& Jayawardhana 2006). These results suggest that the dynamic star-disk interaction probably controls the accretion process in very different mass domains and over a large time span, as long as an accretion disk is present. As discussed in this volume by Robert Mathieu, close binary systems, where circumstellar disks cannot survive, but only a circumbinary disk is present are also seen to accrete with the same characteristics as the stars we nowadays believe to be single (Mathieu, Stassun, Basri, et al. 1997; Alencar, Melo, Dullemond, et al. 2003; Jensen, Dhital, Stassun, et al. 2007). Accretion, as we know it, then manages to proceed in environments with very different physical characteristics too.

The ideal way to study the dynamical star-disk interaction is to follow young star-disk systems for several rotation periods and to look for evidences of variable accretion and outflow events that can be related to time variable funnel flows and other predictions of numerical models. This was done for the CTTS AA Tau at different epochs and the main results are discussed in the following section.

\section{AA Tau: a test case for the dynamical evolution of magnetospheric accretion}

AA Tau is one of the few CTTSs that was studied synoptically with simultaneous photometry and high-resolution spectroscopy covering several stellar rotation periods at different epochs. The 1995, 1999 and 2004 observing campaigns yielded a huge amount of detailed information about the accretion and outflow processes and their connection (Bouvier, Chelli, Allain, et al. 1999; Bouvier et al. 2003; Bouvier et al. 2007).

The AA Tau system presents modulation of photometric and spectroscopic diagnostics with a period of 8.2 days, which is the rotational period of the star. The light curves show quasi-cyclic recurrent minima with very little color changes that were attributed 

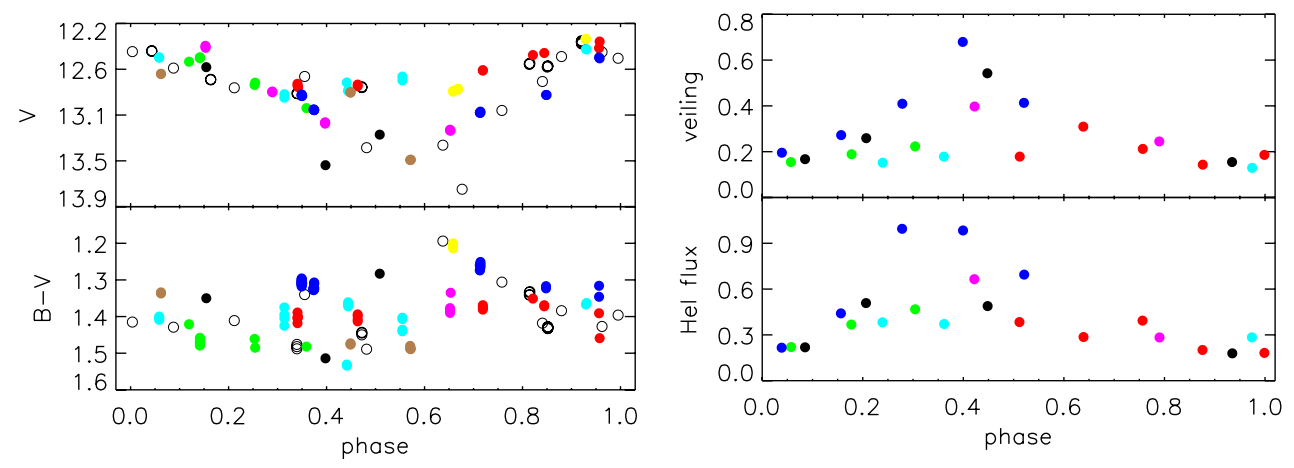

Figure 3. Left: AA Tau V and B-V curves as a function of rotational phase. Light blue and red cycles present shallow photometric minima. Right: AA Tau veiling and HeI $5876 \AA$ line flux as a function of rotational phase. Different colors represent different rotation cycles. Figures from Bouvier et al. (2007).

to obscuration of the central star by the optically thick, magnetically-warped inner disk region located near the co-rotation radius (Fig. 3). The inner disk warp is expected by magnetospheric models to develop due to the interaction of the disk with an inclined (non-axisymmetric) magnetosphere (Terquem \& Papaloizou 2000; Romanova et al. 2003). A blueshifted absorption component is seen all the time in the Balmer lines and is supposed to arise from a cold inner disk wind. The redshifted absorption components of the Balmer line profiles exhibit a periodic modulation at the stellar rotation period with a maximum strength when the main accretion funnel flow passes through our line of sight, which also corresponds to the photometric minima. Redshifted absorption is thought to appear when the hot spot region is seen through the accretion funnel and is therefore a strong accretion diagnostic. The veiling is also modulated by the appearance of the accretion shock and the photospheric and HeI radial velocities present periodic variations attributed to spots (cold and hot) at the stellar surface. All the above characteristics are present in the two observational campaigns that had simultaneous photometric and spectroscopic measurements (1999 and 2004) and give support to the general predictions of magnetospheric accretion models.

The AA Tau system also presented evidence for large scale changes occurring in the magnetospheric structure of the star on timescales of weeks and years. These cannot be explained by static magnetospheric models and require time-variable accretion funnels.

The AA Tau light curve presents cycles of deep and shallow eclipses, where the obscuring screen characteristics are very distinct. Furthermore, the cycles that only show a shallow photometric minimum do not exhibit a very deep redshifted absorption component in the Balmer lines and they present low veiling and HeI $5876 \AA$ line flux values (light blue and red dots in Fig. 3). The HeI 5876 ̊ line in AA Tau is composed most of the time of only a narrow component that is thought to come from a region close to the accretion hot spot (Beristain, Edwards \& Kwan 2001), so like the veiling, it should also trace the physical conditions near the accretion spot. The spectroscopic characteristics described above indicate that accretion is at a very low level when most of the occulting circumstellar material is absent. There is therefore a clear correlation between the accretion level, as measured by spectroscopic features such as veiling and line components related to the accretion shock region, and the formation of the inner disk warp, as measured by the photometric minimum depth, both supposedly depending on the time variable magnetic configuration at the disk truncation radius. 

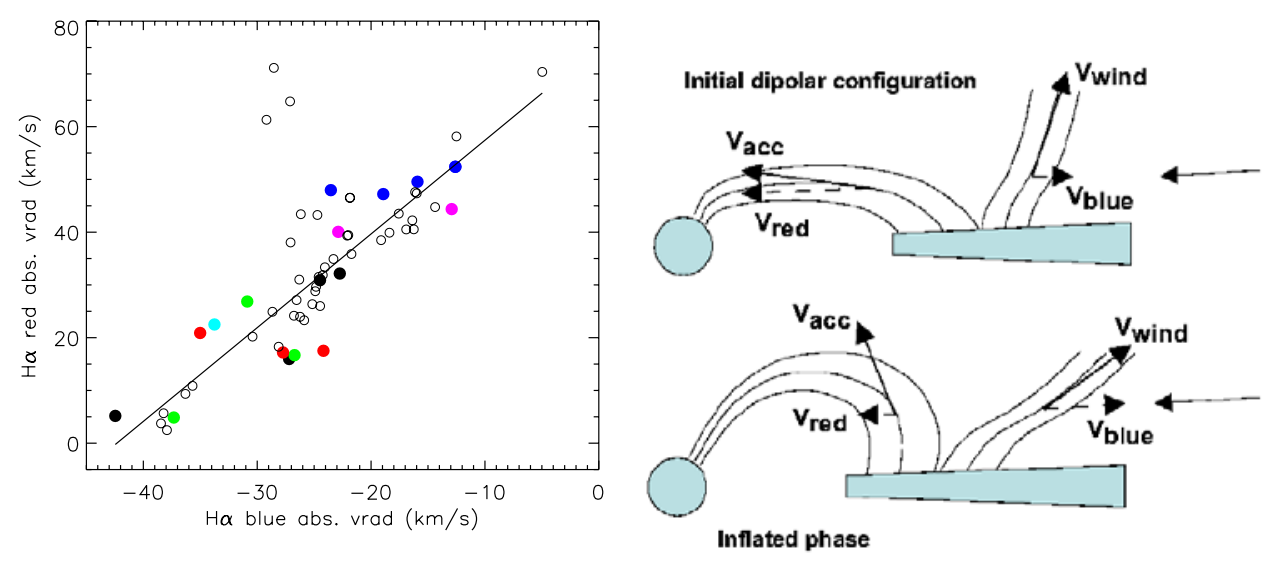

Figure 4. Left: measured relation between the radial velocities of $\mathrm{H} \alpha$ redshifted and blueshifted absorption components. Open symbols correspond to the 1999 campaign and filled symbols to the 2004 campaign. Different colors refer to different rotation cycles. Right: sketch of magnetospheric inflation showing the radial velocities of the blue (wind) and red (accretion) absorption components of $\mathrm{H} \alpha$. The arrow on the right side indicates the line of sight to the AA Tau system (Bouvier et al. 2003; Bouvier et al. 2007).

There is also a very tight correlation between the radial velocities of the redshifted (accretion) and blueshifted (wind) absorption components of the $\mathrm{H} \alpha$ line that was observed in both the 1999 and 2004 campaigns. This provides evidence for a physical connection between time dependent accretion and outflow in CTTSs. It was interpreted by Bouvier et al. (2003) as a first observational evidence of inflation of the stellar magnetosphere caused by differential rotation between the stellar magnetic field lines and the inner disk, which are predicted by numerical simulations of star-disk interaction (see $\S 1$ ). The idea is illustrated in Figs. 1 and 4. Starting from a initial dipolar configuration, differential rotation between the inner disk edge and the stellar surface inflates the magnetic field lines that thread the inner disk region, reducing the accretion flow onto the star. At a certain critical point, the field lines open and reconnect leading to enhanced wind outflow. After reconnection, the initial dipolar configuration is restored. It was proposed by Bouvier et al. (2003) that the projected radial velocity of the redshifted absorption component of $\mathrm{H} \alpha$ measures the curvature of the accretion funnel, as shown in Fig. 4. If the wind traced by the blueshifted absorption component of the Balmer lines originates close to the inner disk edge, the projected radial velocity of this component is expected to decrease as that of the redshifted absorption component increases, thus providing the observed correlation. This is exactly what is observed in AA Tau in both campaigns. Just before and during the shallow eclipses and low accretion cycles the field lines are inflated and opening, as measured by the projected radial velocities of the wind and accretion components. In this phase a hot wind outflow develops, traced by the appearance of a broad blueshifted component in the HeI $5876 \AA$ line (Fig. 5), which is attributed to hot winds according to Beristain, Edwards \& Kwan (2001). After this, the deep eclipses and normal veiling values are restored, as is the initial configuration of the accretion funnel. These results tend to support the idea of magnetospheric accretion cycles on a timescale of several rotation periods in accreting $\mathrm{T}$ Tauri stars, although the periodicity of such episodes as predicted by dynamical models still remains to be tested with longer time series.

AA Tau also presented long term photometric variations. Comparing four observational photometric campaigns from 1995, 1999, 2003 and 2004, Bouvier et al. (2007) showed 

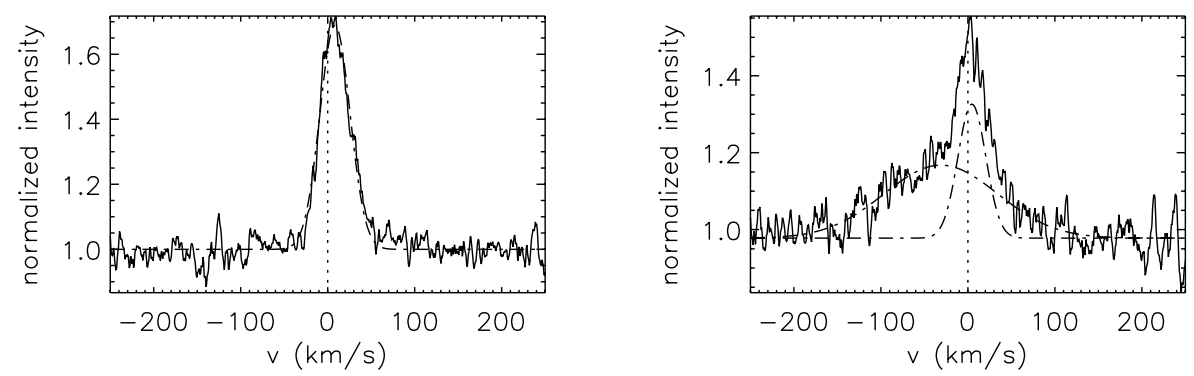

Figure 5. AA Tau observed HeI $5876 \AA$ line (solid lines) and decomposition with gaussians (dash-dotted lines). Left: profile dominated by a narrow component most of the time. Right: narrow and broad blueshifted components observed at the end of a low accretion cycle. Figure adapted from Bouvier et al. (2007).

that the shape and depth of the eclipses can change dramatically in years, although they vary only slightly on a timescale of weeks within each observing campaign. The general characteristics of the light curve is the same in the several observing seasons, all presenting a constant maximum interrupted by episodes of recurring minima with little color change. However, the long term changes of the light curve shape in a timescale of years can be quite important. The light curves can change from a broad very deep eclipse per cycle that does not show much variability amplitude (1995 campaign), to two shallower and variable eclipses per cycle (1999 campaign), back to a major minimum per cycle with variable amplitude (2003 and 2004 campaigns). This indicates that the shape of the obscuring screen can change drastically over the years, which suggests that the stellar magnetic field interaction with the inner disk, that is thought to produce the inner disk warp, varies significantly over the years too.

The AA Tau system showed that the accretion process is variable on several timescales, all of them related to the asymmetric, non-steady magnetospheric configuration. It varies in days, as shown by the veiling changes due to an inclined magnetosphere with respect to the rotation axis, it varies in weeks due to inflation cycles caused by differential rotation between the stellar magnetosphere and inner disk regions and it varies in years, as picture by the variable overall configuration of the inner disk warp that is thought to arise due to the interaction of an inclined magnetic field and the inner circumstellar disk.

\section{Conclusion}

We have shown that magnetospheric accretion models can account for the general observed characteristics of CTTSs. However, axisymmetric steady-state magnetospheric models cannot explain the time variable characteristics of such accreting young systems.

The star-disk interaction was shown to be very dynamical on several timescales (hours, weeks, months and years) and is thought to be mediated by the stellar magnetic field. This dynamical aspect of the star-disk interaction may have implications to the short and long term variability of inflows and outflows and to the angular momentum evolution of the star-disk system.

Finally, in CTTSs, accretion and outflow are strongly related processes and models that try to explain these systems should always take both into account. 


\section{Acknowledgements}

S.H.P.A. would like to acknowledge support from IAU, CNPq and Fapemig.

\section{References}

Alencar, S. H. P., \& Batalha, C. 2002, ApJ 571, 378

Alencar, S.H.P., Johns-Krull, C.M. \& Basri, G. 2001, AJ 122, 3335

Alencar, S.H.P., Melo, C.H.F., Dullemond, C.P., Andersen, J., Batalha, C., Vaz, L.P.R., \& Mathieu, R.D. 2003, A\&SA 409, 1037

Ardila, D.R., Basri, G., Walter, F.M., Valenti, J.A., \& Johns-Krull, C.M. 2002, ApJ 566, 1100

Beristain, G., Edwards, S., \& Kwan, J. 2001, ApJ 551, 1037

Bouvier, J., Covino, E., Kovo, O. et al. 1995, A\&SA 299, 89

Bouvier, J., Chelli, A., Allain, S., Carrasco, L., Costero, R., Cruz-Gonzalez, I., Dougados, C., Fernandez, M., Martin, E.L., Menard, F., Mennessier, C., Mujica, R., Recillas, E., Salas, L., Schmidt, G., \& Wichmann, R. 1999, A\&A 349, 619

Bouvier, J., Grankin, K.N., Alencar, S.H.P., Dougados, C., Fernandez, M., Basri, G., Batalha, C., Guenther, E., Ibrahimov, M.A., Magakian, T.Y., Melnikov, S.Y., Petrov, P.P., Rud, M.V., \& Zapatero Osorio, M.R. 2003, A\&SA 409, 169

Bouvier, J., Alencar, S.H.P., Boutelier, T., Dougados, C., Balog, Z., Grankin, K., Hodgkin, S.T., Ibrahimov, M.A., Kun, M., Magakian, T.Yu., \& Pinte, C. 2007, A 6 A 463, 1017

Calvet, N. \& Gullbring, E. 1998, ApJ 509, 802

Daou, A.G., Johns-Krull, C.M. \& Valenti, J.A. 2006, AJ 131, 520

Donati, J.-F., Jardine, M.M., Petit, P., Morin, J., Bouvier, J., Cameron, A.C., Delfosse, X., Dintrans, B., Dobler, W., Dougados, C., Ferreira, J., Forveille, T., Gregory, S.G., Harries, T., Hussain, G.A.J., Menard, F., \& Paletou, F. 2007, in: G. van Belle (ed.), 14th Cambridge Workshop on Cool Stars, Stellar Systems, and the Sun, ASP Conf. Series, in press

Edwards, S., Hartigan, P., Ghandour, L., \& Andrulis, C. 1994, AJ 108, 1056

Favata, F., Flaccomio, E., Reale, F., Micela, G., Sciortino, S., Shang, H., Stassun, K.G., \& Feigelson, E.D. 2005, ApJS 160, 469

Goodson, A.P., Winglee, R.M. \& Böhm, K.-H. 1997, ApJ 489, 199

Goodson, A.P. \& Winglee, R.M. 1999, ApJ 524, 159

Grankin, K. N., Melnikov, S. Y., Bouvier, J., Herbst, W., \& Shevchenko, V. S. 2007, A\&A 461, 183

Gullbring, E., Calvet, N., Muzerolle, J., \& Hartmann, L. 2000, ApJ 544, 927

Hartmann, L., Hewett, R. \& Calvet, N. 1994, ApJ 426, 669

Herbig, G.H. 2007, AJ 133, 2679

Jardine, M., Cameron, A.C., Donati, J.-F., Gregory, S.G., \& Wood, K. 2006, MNRAS 367, 917

Jayawardhana, R., Coffey, J., Scholz, A., Brandeker, A., \& van Kerkwijk, M.H. 2006, ApJ 648, 1206

Jensen, E.L.N., Dhital, S., Stassun, K.G., Patience, J., Herbst, W., Walter, F.M., Simon, M., \& Basri, G. 2007, AJ 134, 241

Johns, C.M., \& Basri, G. 1995, ApJ 449, 341

Johns-Krull, C.M., Valenti, J.A., Hatzes, A.P., \& Kanaan, A. 1999, ApJ (Letters) 510, L41

Kurosawa, R., Harries, T.J. \& Symington, N.H. 2006, MNRAS 370, 580

Kurosawa, R., Romanova, M.M. \& Harries, T.J. 2007, Star-disk interaction in young stars, IAU Symposium No. 243, poster available at http://www.iaus243.org/

Kwan, J., Edwards, S. \& Fischer, W. 2007, ApJ 657, 897

Long, M., Romanova, M.M. \& Lovelace, R.V.E. 2007, MNRAS 374, 436

Mathieu, R.D., Stassun, K., Basri, G., Jensen, E.L.N., Johns-Krull, C.M., Valenti, J.A., Hartmann, L.W. 1997, AJ 113, 1841

Matt, S., Goodson, A.P., Winglee, R.M., Böhm, K.-H. 2002, ApJ 574, 232

Matt, S. \& Pudritz, R.E. 2005a, MNRAS 356, 167

Matt, S. \& Pudritz, R.E. 2005b, ApJ (Letters) 647, L45

Montmerle, T., Grosso, N., Tsuboi, Y., Koyama, K. 2000, ApJ 532, 1097

Muzerolle J., Hartmann L. \& Calvet N. 2001, ApJ 550, 944 
Oliveira, J.M., Foing, B.H., van Loon, J.T., \& Unruh, Y.C. 2000, A\&A 362, 615

Petrov, P.P., Gullbring, E., Ilyin, I., Gahm, G.F., Tuominen, I., Hackman, T., \& Loden, K. 1996, $A \mathscr{E} A 314,821$

Reipurth, B. \& Aspin, C. 2004, ApJ (Letters) 608, L65

Romanova, M.M., Ustyugova, G.V., Koldoba, A.V., \& Lovelace, R.V.E. 2002, ApJ 578, 420

Romanova, M.M, Ustyugova, G.V., Koldoba, A.V., Wick, J.V., \& Lovelace, R.V.E. 2003, ApJ 595,1009

Romanova, M.M., Ustyugova, G.V., Koldoba, A.V., \& Lovelace, R.V.E. 2004a, ApJ (Letters) 616, L151

Romanova, M.M., Ustyugova, G.V., Koldoba, A.V., \& Lovelace, R.V.E. 2004b, ApJ 610, 920

Scholz, A., \& Jayawardhana, R. 2006, ApJ 638, 1056

Shu, F., Najita, J., Ostriker, E., Wilkin, F., Ruden, S., \& Lizano, S. 1994, ApJ 429, 781

Shu, F.H., \& Shang, H. 1997, in: B. Reipurth \& C. Bertout (eds.), Herbig-Haro Flows and the Birth of Stars, IAU Symposium No. 182 (Kluwer Academic Publishers), vol. 182, p. 225

Stempels, H.C. \& Piskunov, N. 2002, A\&A A 391, 595

Symington, N.H., Harries, T.J., Kurosawa, R., \& Naylor, T. 2005, MNRAS 358, 977

Terquem, C. \& Papaloizou, J.C.B. 2000, A\&A 360, 1031

Tsuboi, Y., Imanishi, K., Koyama, K., Grosso, N., \& Montmerle, T. 2000, ApJ 532, 1089

Uzdensky, D.A., Königl, A. \& Litwin, C. 2002, ApJ 565, 1191

Valenti, J.A. \& Johns-Krull, C.M. 2004, ApESSS 292, 619

von Rekowski, B. \& Brandenburg, A. 2004, A\& A 420, 17

von Rekowski, B. \& Brandenburg, A. 2006, Astron. Nachr. 327, 53

\section{Discussion}

EDWARDS: We don't normally see CTTS light curves with deep recurrent minima like the ones observed in AA Tau. Is there anything special about this system that makes it more likely to have an inner disk warp that eclipses the stellar photosphere ? Is the inclination of the magnetic axis with respect to the rotation axis particularly large ?

AlENCAR: In a recent paper by Grankin et al. (2007), they show that only about $15 \%$ of the CTTSs in their sample exhibit light curves similar to AA Tau. This is indeed a small number of objects and the authors suggest that this could be related to an inclination effect. Only systems seen at high inclination with respect to the line of sight, which is the case of AA Tau, would exhibit a light curve pattern related to the occultation of the photosphere by circumstellar material from the disk. Regarding AA Tau specifically, Kurosawa and collaborators also had to use a large inclination $\left(>60^{\circ}\right)$ of the magnetic axis with respect to the rotation axis to try to qualitatively reproduce the observed time variable $\mathrm{H} \beta$ line with the magnetospheric configuration and physical parameters of the Romanova et al. (2003) 3D MHD simulations. However, Bouvier et al. (2007) estimated the tilt between the axis of the large scale magnetosphere and the rotation axis to be around $20^{\circ}$ based on the modeling of radial velocity variations of the HeI $5876 \AA$ line, supposed to be due to a major hot spot at the stellar surface. Valenti \& JohnsKrull (2004) derived an inclination of $12^{\circ}$ between the two axis from spectropolarimetric measurements. So it is not clear if the inclination of the magnetic to the rotation axis is very large in AA Tau and I would say that so far there is no conclusive indication that AA Tau is a special system among CTTSs. It is apparently just a system seen at high inclination with respect to the line of sight.

Someone: How do the observed line profiles compare with those calculated from the magnetospheric configuration of the Romanova MHD simulations ? 
Alencar: The first time I saw emission line profiles calculated using the density, temperature and geometric structure of the 3D MHD simulations was in this conference in the poster by Ryuchi Kurosawa. I talked to him and they have just started to compare general features of theoretical and observed line profiles for AA Tau. Some characteristics are well reproduced by the model profiles, like the overall scale of variability across the $\mathrm{H} \beta$ line, but the theoretical profiles do not reproduce other observed fearures, like the absorption near the $\mathrm{H} \beta$ line center. They plan to do a direct comparison between observed and calculated line profiles soon.

JOHNS-KRULL: I would like to add a comment. We published time series spectroscopic observations of T Tau, which is a CTTS viewed pole-on, as is TW Hya which was discussed in the talk. T Tau also displays strong emission line variability, indicating that the mass accretion rate is variable on this star on short timescales as well.

SHU: We must interpret with care the importance of dynamical predictions of numerical simulations, since the simulations still run over a small timescale compared to the PMS evolution.

AlENCAR: I agree. The simulations typically run only over a few hundred (thousand ?) Keplerian periods at the inner disk edge.

Romanova: I disagree. We have reached a steady state in our simulations. So our predictions are robust.

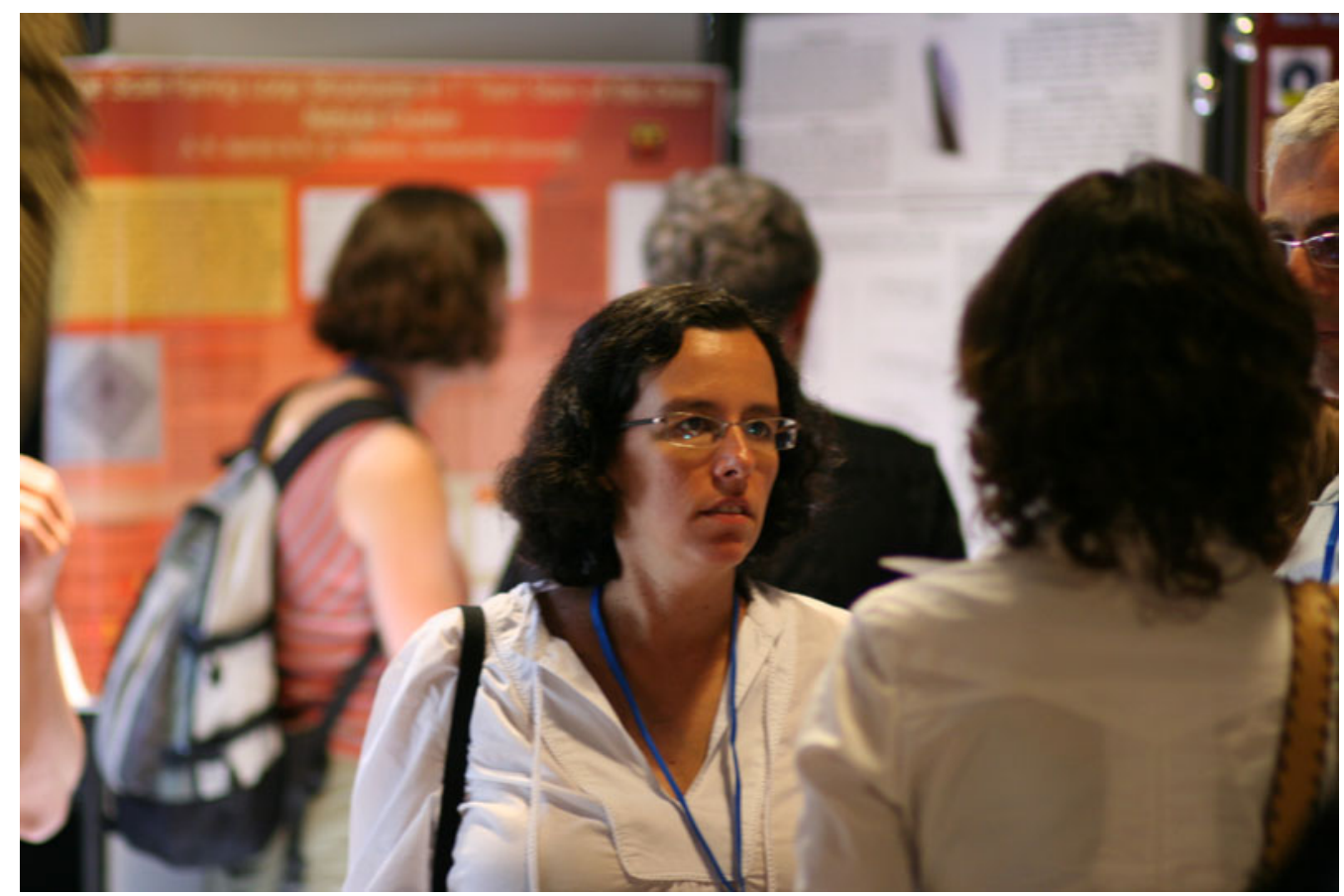



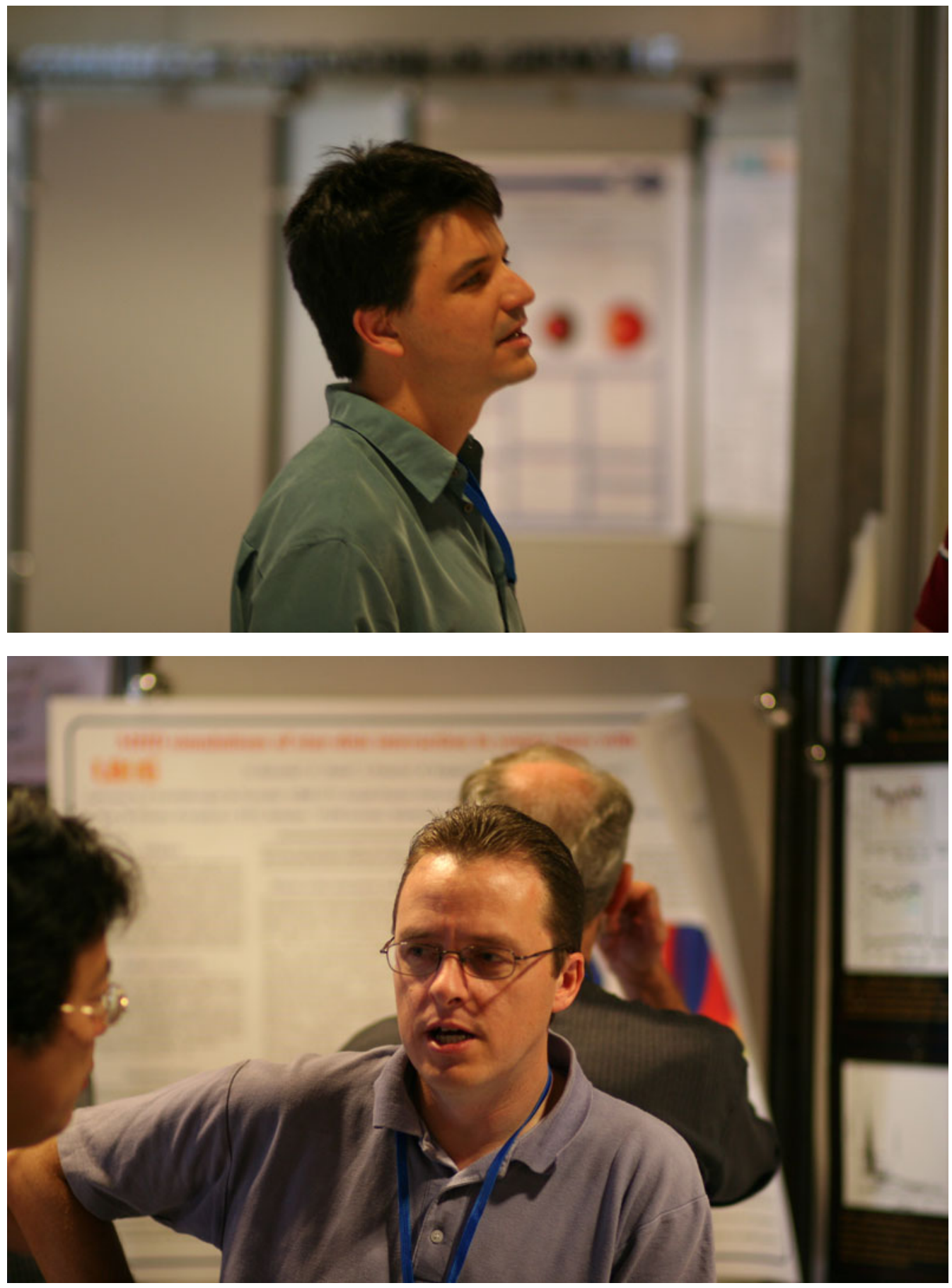